\title{
High Performance Polymers in Additive Manufacturing Processes: Understanding Process, Structure and Property.
}

\author{
Manuel Garcia-Leiner ${ }^{1}$, Daniel P. Dennies ${ }^{2}$ and Atif Yardimci $^{3}$ \\ 1. Exponent, Inc. Polymer Science and Materials Chemistry, Bowie, MD, USA. \\ 2. Exponent, Inc. Materials and Corrosion Engineering, Irvine, CA, USA. \\ 3. Exponent, Inc. Biomedical Engineering, Chicago, IL, USA.
}

Additive Manufacturing (AM), otherwise known as three-dimensional (3D) printing, is a growing technology area comprised of a spectrum of processes that allow production of three-dimensional solid objects of virtually any shape from information obtained from a digital object. These days, AM processes are driving major innovations in multiple areas, such as engineering, manufacturing, art, education and medicine. In its broadest sense, AM processes use additive approaches where materials are applied in successive layers in order to produce a final part, differing from traditional subtractive manufacturing techniques that often rely on the removal of materials by methods such as cutting or milling. AM processes are not necessarily new. They were introduced commercially in the early 90's for the manufacture of complex metal parts and have almost a 30-year history for plastic objects, mainly due to prototyping efforts that drove the development of multiple commercial products using techniques from stereo-lithography to laser based powder based fusion processes.

The global market size for AM products is expanding at a rapid pace. Even though the manufacturing costs for AM remain higher compared to conventional processes, significant reduction in efficiency and logistics in the coming years would make AM approaches attractive for specific cases, especially through reduction of tooling costs, design freedom and reduction in assembly requirements. _High demanding applications such as medical, aerospace, oil and gas exploration, military and defense and semiconductor applications will benefit directly from the expected growth of AM processes.

A growing number of polymeric resins are becoming available due to developments of new processes and technological advancements in AM. Specifically, high-performance thermoplastics are perhaps the most promising material candidates for the adoption of AM into high demanding engineering applications. Because of this, the fundamental understanding of the AM process physics, as well as the resulting structure of high-performance thermoplastics and its relation to their performance in critical environments is crucial for the development of new technologies and complex processing techniques.

In this regard, we provide a series of examples where polymeric systems are used for the production of parts for high demanding applications using various AM processes. In particular, this work describes an in depth study of the morphological changes observed in selected high performance polymer resins when subjected to conditions typically observed in common AM processes, including powder bed fusion processes such as Selective Laser Sintering (SLS), as well as extrusion-based approaches such as Fused Deposition Modeling (FDM).

In this study, we use selected poly(etherketoneketone) (PEKK) resins that due to their superior properties (including extremely high thermal properties and polymorphic crystalline nature, superior mechanical properties, chemical resistance and low flammability) represent a viable material choice for various high demanding engineering applications. We focus on the analysis of the crystal structure and 
the development of polymorphism in PEKK systems (Figure 1) under conditions often encountered in SLS and FDM processes. We observed that parts produced through various processes and conditions will display different crystalline morphology that will lead to changes in their mechanical properties (i.e. tensile strength and elongation at break) (Figure 2). We conclude that the understanding and precise control over the morphological changes in these materials during AM processing is critical for their successful introduction into high demanding engineering applications.

\section{References:}

[1] KH Gardner et al, Polymer 33 (1992), p. 2483-2495.

[2] ZD Cheng et al, Macromol. Chem Phys. 197 (1996), p. 185-213.

[3] EA Klop et al, Journal of Polymer Science: Part B: Polymer Physics 33 (1995), p. 315-326.

[4] F Muller et al, Unites States Patent 8,299,208 B2, (2012).

[5] C Bertelo et al, World Patent Application WO2012/047613 A1, (2012).

[6] C Bertelo et al, United States Patent Application US2013/0323416, (2013).
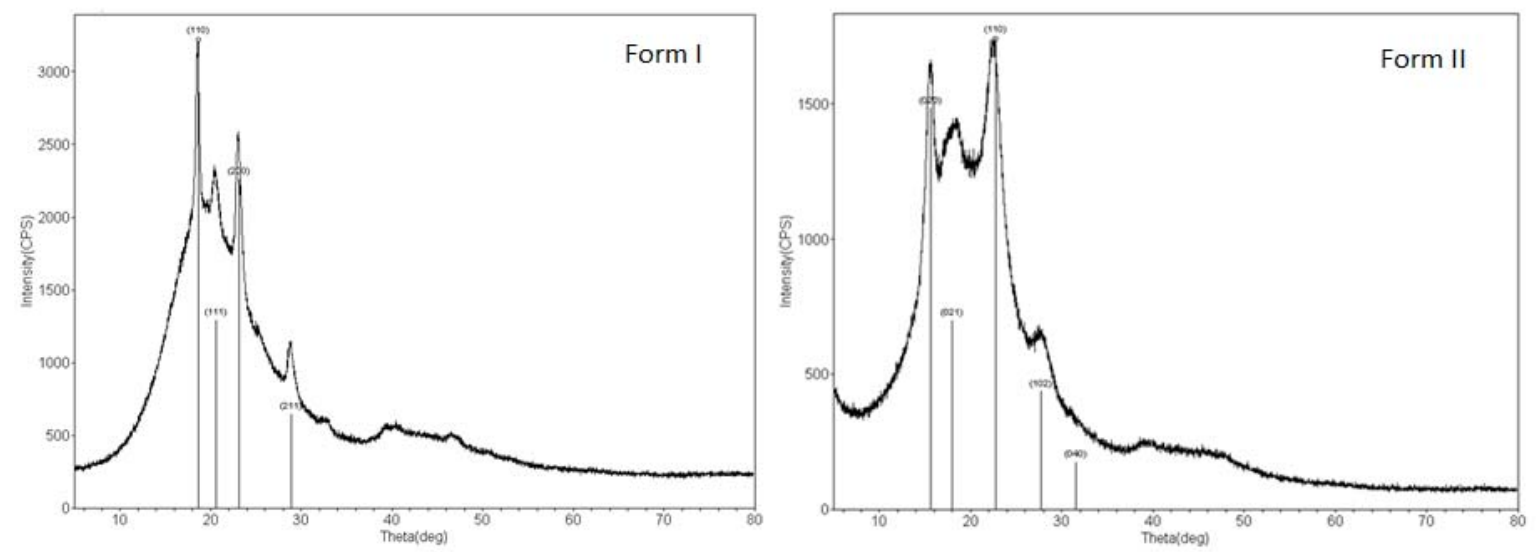

Figure 1. Wide Angle X-ray Diffraction (WAXD) patterns for PEKK crystalline polymorphs (Form I and Form II). The presence of these polymorphs will depend on the specific AM process used.

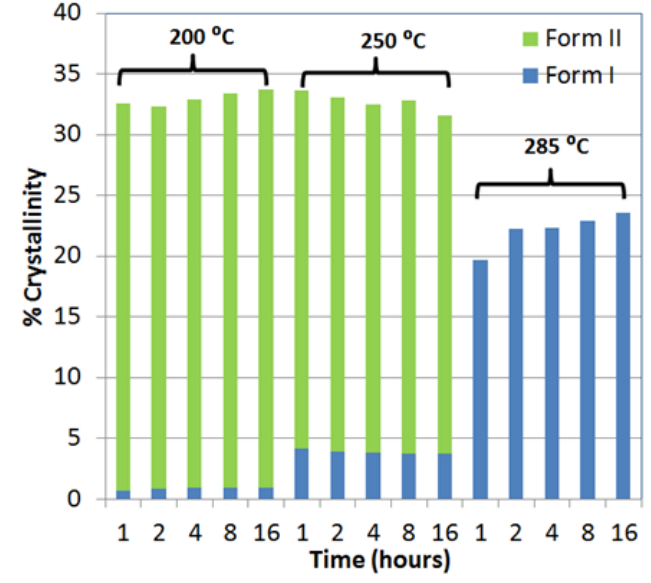

(a)

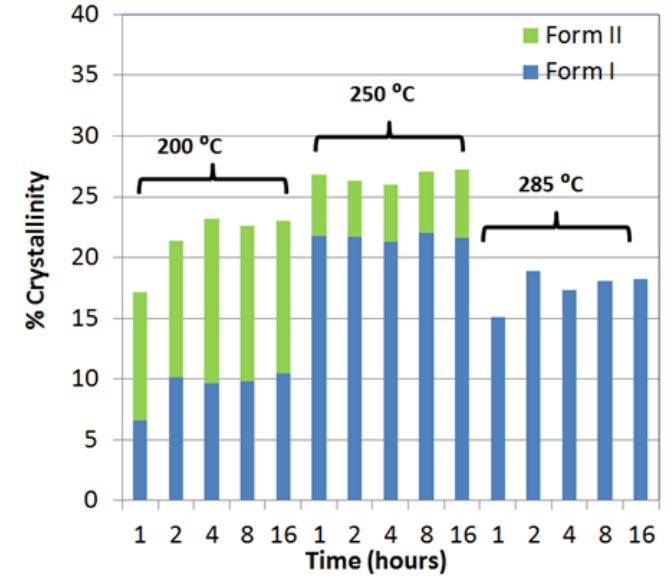

(b)

Figure 2. Influence of processing conditions on the crystal composition in PEKK parts produced by various AM processes. a) Powder bed fusion processes (SLS); b) Extrusion-based processes (FDM). 